\title{
CAREGIVING AND ELDERLY HEALTH IN MEXICO
}

\author{
Antonio J. Trujillo, Thomas A. Mroz, Claudia Piras, Gustavo Angeles, and Nhan Tran
}

\section{Abstract}

Using the National Mexican Health and Aging Study panel dataset, the authors estimate the effect of having informal care on the probability of dying and on the change in elderly health over a twoyear period. Three measures of functional health were used: self-reported health, activities of daily living, and instrumental activities of daily living. We develop an empirical strategy that relies on the panel structure of the dataset to sort out the possible correlation between unobservable characteristics that affect both elderly health and an individual's decision to provide informal care. Our findings suggest that informal care provided by daughters reduces the probability of dying. In addition, informal care provided by daughters reduces the probability of having a decline in activities of daily living and instrumental activities of daily living, while it has no effect on the observed changes in self-reported health status. The protective effect of informal care provided by sons is not statistically significant for any health outcomes. A discussion of the policy options to increase elderly health and to improve the role of caregivers is included.

\begin{abstract}
Mexico, like most developing economies in Latin America, does not escape the challenges that a rapidly growing elderly population imposes on all members of society. These challenges include how to provide seniors with financial assistance, formal and informal social support, and health care (1). To highlight two aspects of the aging process in Mexico, from 1930 to 1995 mortality risk declined by $60 \%$ and fertility rates have declined continuously since 1960 . The combination of both trends will propel a rapid increase in the elderly population, from $4.4 \%$ of the total population in 2000 to $25 \%$ in 2050 . This demographic transformation is occurring in a period when both infectious diseases and chronic conditions are prevalent, a process the authors refer to as "epidemiology polarization." Changes in patterns of morbidity and mortality among the elderly result from this polarization. In particular, it implies a higher rate of disabilities, more functional limitations, and an increase in medical care consumption among seniors greater than those projected in developed societies during similar demographic periods. How society shares the economic cost of this aging process will shape Mexico's present and future growth.

Some of the policy options for handling the increasing costs of an aging population could involve shifting some of the caregiving responsibility to families. The potential transition from formal to informal care may reduce the financial burdens of health care expenditures on the public sector. However, these potential savings need to be weighed against the impact of informal care on the health of the elderly and on the caregiver. In addition, a caregiver's
\end{abstract}


decision to terminate or reduce participation in the labor force is an issue that needs to be considered to assess the overall benefits to the economy.

This study examines how the availability of informal care influenced mortality and changes in health among seniors in Mexico from 2001 to 2003. In addition to mortality information, three measures of functional health status were used: self-reported health status (SRHS), activities of daily living (ADLs), and instrumental activities of daily living (IADLs). This analysis also explores whether care provided by daughters and sons during this period might have had heterogeneous effects on the health of the elderly. The period of the study is of particular interest because of the accelerated aging process in Mexico since 2000.

It is important to note that this research does not compare the relative health benefits of informal and formal care, nor will it test the cost-effectiveness of either. Instead, we are testing whether people who receive informal care have better health prospects than those who do not. This research should be considered an initial, but necessary, step toward an assessment of the benefits of informal care on the health of the elderly.

Research on aging has pointed out the relevance of informal care on mortality, changes in health, and medical expenditures. Most of the growing evidence relates to developed countries. This paper contributes to the existing body of literature on informal care in three fundamental ways.

First, the authors evaluate how informal care affects elderly health and mortality in the context of a developing economy, rather than estimating how it affects formal medical care expenditures. Most of the existing body of literature deals with the replacement of formal with informal care and its relevance for public finance. This literature also includes how informal care influences caregivers' health, labor force participation, and retirement conditions. For instance, if the need to care for the aging population draws women out of the labor market, over time this may hurt their ability to save for retirement. The overall inability of these societies to sustain economic growth in the future may place them in jeopardy. Additionally, human capital deterioration—including mental health and medical costs - may result from the stress of providing care. This is cause for serious concern (2). Women who provide informal care may have a markedly higher level of depression and, in general, a lower level of well-being than men (3). Nevertheless, assistance for the elderly may also have a positive influence upon the life of caregivers (4).

Second, our analysis suggests important insights into assessing the overall impact of informal care upon the health of seniors in developing countries. Caregivers may influence the health of seniors through different channels. Caregivers may help the elderly with selfmanagement of chronic conditions and preventive efforts. Caregivers may assist the elderly in their daily lives in such a way that, over time, improves their functional health. Caregivers may influence, directly or indirectly, the external environment, which can prevent accidents and subsequent increases in disabilities or mortality among seniors.

Services provided by caregivers may have little or no impact on elderly health, or they may even have negative consequences. For instance, caregivers may provide poor care to the elderly, particularly for those with chronic conditions. Caregivers may over-protect the 
elderly in their daily lives and diminish their functional independence and capacity. Last, caregivers may alter the environment to favor other members of the household, inflicting unnecessary risks for seniors. In sum, the net effect of informal care may be insignificant or even harmful; therefore, it becomes an empirical question that should be tested.

Third, the National Mexican Health and Aging Study (MHAS) provides us with a unique opportunity to explore this issue, since available panel datasets are uncommon in developing countries. This information allows the authors of this study to partially control for endogeneity between an individual's decision to provide informal care and elderly health. It is important to note, however, that it is difficult to provide a simple definition of a "causal effect" of caregiving on elderly health, as it is nearly impossible to envision how one might cause a woman to care for her parents. Given that an experimental design is not feasible, researchers must employ methods using observational data to gauge the magnitude of the causal effect.

In this study, our identification strategy was to estimate the unbiased effect of informal care on elderly health that relies on the use of a Lagged Dependent Variable Model (LDVM) where previous health, as well as observable covariates at the baseline point, are used to explain changes in health during the study period. Although we explored the possibility of using a difference-in-difference approach or instrumental variable estimates using pool data, the results using a LDVM were more robust, given the lack of variability in some covariates within individuals. We provide further clarification of this issue in the next section of the paper.

Last, the authors estimate the heterogeneous effect of informal care provided by sons and daughters. Different hypotheses have been advanced to explain the gender gap in the provision of informal care for the elderly $(5,6)$. Each of these hypotheses is likely to be a factor in understanding an individual's decision to provide care to a senior and its impact upon the health of the elderly. For instance, as women who work are less able or less willing to care for the elderly, more private and government expenditures may be needed to substitute for this informal care. These decisions may influence seniors' health.

Alternatively, one could foresee a greater role for men as caregivers within families as a consequence of family structure changes driven by demographic transitions in Mexico. Therefore, it is important to understand the key differences between daughters and sons who provide care for the elderly and their impact on seniors' health.

In the next section, we review the relevant literature on gender differences in caregiver activities and their impact upon health, followed by a description of the MHAS dataset and construction of variables. We then present the conceptual framework and empirical results.

\section{BACKGROUND LITERATURE ON CAREGIVING}

In this section, the authors briefly discuss and summarize two important aspects of the existing literature on informal care: the effects of care on health and gender differences in providing that care. We do not review the literature on the substitution effect between formal and informal care (7) related to the burden of informal care upon caregivers. The purpose in this section is to delineate the primary conceptual arguments to justify the links between 
informal care and health and to support the reasons behind differential effects of informal care provided by females and males.

From a conceptual point of view, the connection between seniors' health and informal care has received limited attention. Clark (2003) highlights the relevance of the social environment in which the elderly manage their own illnesses (8). According to Clark, direct and indirect links from informal care can influence an individual's ability to manage a chronic illness and, in time, the health of the elderly (9). Direct effects may include helping the elderly with specific tasks such as glucose monitoring and organizing or taking prescriptions. Indirect tasks may include verbal support and sharing experiences.

In addition, caregivers may shape the external environment in a way that can facilitate or hinder an elderly person's life. For instance, caregivers may help the elderly follow certain diets or reduce hazardous elements within the household. Moreover, Antonucci and Jackson (1987) suggest that supportive interactions from caregivers can boost health-promoting behavior (10). In sum, the current literature shows evidence of a modest, positive relationship between social support and self-management behaviors for illnesses (11). However, as Gallant, Spitze, and Prohaska (2007) indicate, the body of literature in this area is small and generally applied to specific diseases (11).

A review of the literature suggests that females are more likely than males to provide informal care to their elderly parents (see, for example, Finley, 1989; Iacovou, 2000; Lee et al., 1993; Neal et al., 1997; Sarkisian and Gerstel, 2004) (5, 6, 12-14). Five hypotheses have been advanced to explain the gender gap in the provision of informal care for the elderly: the cost of time or time-availability hypothesis, the socialization hypothesis, the external resources hypothesis, the specialization-of-task hypothesis, and the gender of parents hypothesis $(5,6)$. Each of these hypotheses is likely to be a factor in explaining an individual's decision to provide care to an elderly family member and could be used as a conceptual framework to explain why females are more likely than males to provide informal care. In an extensive review of the literature, Pinquart and Sorensen (2006) reviewed these hypotheses and reported that females provide more caregiving hours, help with more caregiving tasks, and assist with more personal care than males (3). Thus, it becomes pertinent to explore how informal care provided by females and males may influence the health of the elderly.

Most recently, two additional hypotheses have been suggested to explain gender gaps in caregiving activities. First, there are differential gains for caregivers from taking care of the parents. Elderly parents contribute a return to their children in exchange for their offspring's caregiving activities. If this is the case, sons and daughters may receive different marginal benefits from taking care of aging parents. Variation in the amount and types of care may be due to differences in the value of these marginal gains. The second hypothesis, the principle of substitution, assumes that the choice of caregiver follows a hierarchical pattern. The established pattern of care is that the older adult's spouse will be the primary caregiver, followed by an adult daughter as secondary caregiver in the absence of the spouse. Without the availability of either, another family member assumes primary caregiver responsibilities. Elderly individuals without family members must rely on community aid. 
These hypotheses come primarily from sociology and social work. Yet, during the past 10 years, the economic literature has developed strategic models of bargaining within the family unit to predict a child's decision to provide care to an elderly parent $(15,16)$. Interestingly, most of the predictions from these economic models fit some of the previously stated hypotheses.

On the empirical side, the limited availability of long-panel data and the difficulty of finding good instruments to control for the endogeneity of some of the child and parent variables have been major challenges to understanding the dynamics behind a child's decision to care for an elderly parent. Stern (1995), using panel data for the United States, verifies some of these hypotheses after controlling for the endogeneity of such child variables as distance and working status. His findings suggest moderate to low effects of elderly sex, age, race, health, caregiver sex, and marital status (17). Yet, parent marital status and child distance from the parent's home have a large effect on the decision to provide care. After controlling for endogenous factors, work status is no longer significant in the child's decision to provide care (17).

In this article, we tested whether informal care provided by females may have different impacts on the health of the elderly. Despite the relevance of these issues, there are few analytical papers that explore the links between the health of elderly populations and the role of caregivers.

\section{DATA SET, MEASURES, AND METHODS}

\section{Data Set}

The MHAS is a panel database that collects information about the health status and health conditions of Mexican populations 50 years of age and older during two periods, 2001 and $2003^{1}(1,18)$. The survey is nationally representative. The weighting procedure used is fully described in the User's Manual for the MHAS databases. In addition, the sampling design accounts for potential problems of under-representation in the final sample of individuals over 80 years of age, as well as institutionalized individuals. The information included in the survey is similar to that provided by the Health and Retirement Survey in the United States.

Key features of the MHAS survey make it particularly suitable for answering the questions posed in this article. First, it records different dimensions of elderly health for both periods of the study. Second, the survey contains detailed information describing the provider of informal care for the elderly. Third, the survey records a complete battery of variables for both years that allow us to establish traits of socioeconomic, demographic, and past baseline health conditions of the elderly in Mexico. Last, the survey collects full information about individuals who died between 2001 and 2003.

The panel nature of the data provides advantages that previous researchers, using crosssectional data in this area, did not have. Repeat measures for 2001 and 2003 from the same

\footnotetext{
${ }^{1}$ The MHAS Web page (www.mhas.pop.upenn.edu) lists complete information on the agencies and researchers who participated in this project. The data in this article could be directly downloaded from this source. Wong, Diaz, and Higgins (2006) and Pagan, Puig, and Soldo (2007) have used this dataset and provided a further description of it.
} 
subjects gave us the possibility of looking at how changes in health during the study period depend on the availability of informal care in the baseline year after controlling for health status and observable covariates in the baseline year. In this way, part of the correlation between unobservable characteristics and informal care can be eliminated once one controls for an individual's previous health. Unfortunately, the MHAS has only two waves; therefore, we cannot assess how changes in the independent variable over time (availability of informal care) affect subsequent changes in health. Yet, this study results in a stronger statistical inference about the dynamical relationship between informal care and health changes than one could obtain using cross-sectional information.

For the purpose of this study, the authors selected individuals age 60 and older who completed both interviews. In addition, individuals older than 60 in 2001 who died between both periods were analyzed. We used baseline information of the elderly in 2001, including availability of informal care, and related this information to observed changes in health between 2001 and 2003. Our sample in Wave 1 includes 7,171 seniors; among this sample, 450 elderly died during the interview period. The database allows us to construct the relevant information in both waves for 6,811 seniors (including those who died). This forces us to exclude 360 observations, which represent $5 \%$ of the initial sample size. Finally, MHAS added additional seniors during the second round of data collection; however, given that our empirical strategy involved the use of baseline information, we were not able to take advantage of these observations.

\section{Measures}

The analysis incorporates several dimensions of individual changes in health as dependent variables during the period 2001-2003. First, SRHS has been widely recognized as a simple but comprehensive indicator of an individual's health and as a good predictor of an individual's mortality (19). Additionally, self-reported physical functioning and the ability to perform personal care (ADL and IADL) have been widely used to study the health of populations in developed and developing societies (20).

We coded SRHS as 5 if an adult reported in excellent health, 4 in very good health, 3 in good health, 2 in fair health, and 1 if an adult reported in poor health. ADL is an indicator from 0 to 5, where 0 equals the worst condition; IADL is an indicator of functional mobility from 0 to 15, where 0 is also the worst condition. ADLs include activities such as bathing, dressing, toileting, walking, getting in and out of a bed or chair, and eating. IADLs include preparing meals, shopping, managing money, using the telephone, doing light housework, and doing heavy housework, among others. ${ }^{2}$ Each health condition refers to whether the doctor or nurse told an individual that he or she had the health condition in the past. Our study quantifies the changes in each health indicator during the study period and creates a dummy variable that equals one for individuals who had a decline in health and zero for the rest of the sample.

Of particular relevance for this analysis are the survey questions regarding caregivers' characteristics and the activities they provide for elderly relatives. The survey captures the

\footnotetext{
${ }^{2}$ Please see the MHAS questionnaire for a full description of the activities included in the ADL and IADL measures.
} 
financial and non-financial help that daughters and sons provided to elderly parents. This analysis combines the financial and non-financial help. In particular, we combine in one dummy variable for each gender the following two questions in the baseline survey: (a) In the last two years, have you (and/or your spouse) given financial assistance to your parent(s)? Include help to pay costs such as rent; exclude shared housing or shared meals. (b) In the last two years, have you (and/or your spouse) helped your parents with basic personal activities such as dressing, eating, or bathing because of a health problem? Exclude help with household chores, errands, and transportation. One limitation of our study is that we were not able to identify the help provided by members outside the household. However, we believe this is not a common practice in the context of Mexico.

Last, the control variables include information on the societal, cultural, environmental, and biological risk factors affecting an individual's changes in health outcomes. For convenience, the vector of control variables includes demographic and socio-economic characteristics, family support characteristics, and baseline health status.

\section{A Snapshot of the Elderly Population}

Table 1 presents the descriptive statistics of a gender comparison in health by age groups in 2001. Table 2 compares the elderly who died during the study period to those ones who were alive in 2003.

In the baseline year, ADL and IADL indicators were higher (i.e., in better condition) for males than for females in all age groups, despite the fact that the self-reported health status for males and females were similar (see Table 1). Although not shown here, the results for 2003 were similar. In general, there is clear evidence to suggest that females are more likely than males to suffer from hypertension, diabetes (with the exception of individuals older than 85), and lung conditions.

In both years, females were more likely to report disabling conditions (see Table 1). The index of depression declined with age, but the level declined more for females. In both years, females were more likely to report a fall in the last 12 months. In sum, female seniors reported lower functional health status and a higher prevalence of all disabling conditions than male seniors.

Saab (2002) reported better health status of elderly males (21). Several factors may account for this fact. A sample selection factor can provide one tentative explanation for these results. In particular, fewer males than females surpassed the age of 60. Consequently, those males who survive were, on average, healthier than surviving females. Males may be less likely to report health problems and seek care than females. In addition, bias in reported functional health may differ by gender.

Looking at the causes of death among the 450 individuals age 60 or older who died during the study period, this study finds that heart disease is the leading cause of death among male seniors. Heart disease is the second most common cause of death among females. A similar percentage of males and females died from stroke. As expected, a significant number of 
females reported hypertension or experienced a fall in the last month before death. These data are available upon request from the main author.

In addition, as Table 2 reports, males who died were older, less likely to be married, and had fewer children alive. A similar pattern occurred for female elderly. For both males and females, the socio-economic conditions of those who died were lower than those alive. For instance, individuals who died during this period reported lower literacy rate, fewer years of education, and total net wealth lower than individuals who were alive in 2003 (see Table 2). Interestingly, females who died reported higher family support (both household members and females in the household) than those who stayed alive. This may be a consequence of the selection process because females with lower health chose to live with their relatives. This highlights the relevance of controlling these factors using the advantage of the panel information to gauge a caregiver's effect on the probability of dying among both male and female elderly.

Last, Table 3 provides a gender comparison of demographic, socioeconomic status, family support, and baseline health status of the sample in 2001. The number of people married declines with age for both male and female elderly. Socioeconomic indicators, such as literacy, education, and net wealth, decline with age for each gender, while males report better socioeconomic conditions than females. In addition, indicators for family support decrease with age for males, while females seem to report higher family support than males for the older age groups. In sum, all these variables have an impact on the change of individual health observed during the study period. This needs to be considered to isolate the direct effect of having informal care in 2001 on elderly changes in health in the following two years. The model this analysis proposes in the next section serves to measure this effect.

\section{Empirical Model}

We index the $\mathrm{N}$ individuals in the study $i=1, \ldots, N$ subjects; for each individual, information on health and other characteristics is available for two periods. Using a Lagged Dependent Variable specification (LDVM), an individual's health status in the second year could be expressed as:

$$
y_{i 2}=\beta_{0}+B_{1} x_{i 1}+B_{2} y_{i 1}+\varepsilon_{i 1}
$$

where $\mathrm{e}_{\mathrm{i}}$ represents the time-variant component of the error term; the two groups to compare are $\mathrm{x}_{\mathrm{i} 1}=0$ (if informal care is not available in 2001) and $\mathrm{x}_{\mathrm{i} 1}=1$ (if informal care is available in 2001); and $y_{i 1}$ represents the health of an individual in the baseline year.

The difference in health between both years (i.e., the change in score) for an individual $i$ could be denoted as $\mathrm{di}=\mathrm{y}_{\mathrm{i} 2}-\mathrm{y}_{\mathrm{i} 1}$. Therefore, a regression model for the change in health is given by the following equation:

$$
d i=\beta o+B_{1} x_{i 1}+\varepsilon_{i}
$$

This model will allow us to test whether the average effect of informal care in 2001 is zero or positive on an individual's changes in health. Yet, estimating $B_{1}$ using this model is 
biased, due to correlation factors between $x_{i 1}$ and the error term. For instance, a selection process can imply that elderly people with a caregiver are sicker (or more prone to be sick) than elderly people without a caregiver. As a consequence, a negative estimate from this equation can imply this selection process rather than the effect of informal care on elderly health. Thus, the model can be extended as follows to purge potential correlation due to time-variant factors between observable and unobservable characteristics:

$$
d i=\alpha o+\alpha_{1} x_{i 1}+\alpha_{2} z_{i 1}+\alpha_{3} y_{i 1}+\mu_{i}
$$

where $\mu$ i represents the new error term. In this case, $a_{1}$ allow us to test whether informal care is effective in terms of changes in health conditional on initial health ${ }^{3}$ and on demographic and socioeconomic conditions. Vector $z_{i 1}$ includes these variables. Notice that by using the LDVM specification, we eliminate some of the contemporary correlation between unobserved, time-invariant factors that influence both the decision to provide informal care and elderly health. Equation 3 is similar to the framework presented by Hedeker and Gibbons (2006) called analysis of covariance (ANCOVA) on change in score adjusting for pre-treatment effect (22). It is important to notice that this approach differs from first-difference models where the researcher controls explicitly for time-invariant covariates.

In sum, since a panel database for two periods was available, our identification strategy to solve the problem of selection into caregiver activities relies on the use of previous health and time-variable, observable covariates at baseline. Two additional alternatives to deal with the endogeneity of the caregiver, using the panel database that could have been used, are: $(a)$ difference-in-difference approach or $(b)$ search for good instrumental variables. Since we have only two waves of data, with lack of variation in some covariates, the results from difference-in-difference were unstable. We tried several instrumental variables commonly used in the literature (e.g., parents' education, distance of the caregiver from parents' house, number of siblings), yet the results from LDVM were more robust.

In the author's estimation, the index di is a dummy variable that equals one if the individual died during the period and equals zero if he or she was alive in 2003. For the rest of the health indicators, similar dummies were created; for example, SRHS equals one if it declines or zero otherwise. For each health indicator (SRHS, ADL, and IADL), this analysis runs the models including both the people who died and individuals who have a decline in health, as well as eliminating from the sample the people who died. We also run the models for seniors with specific chronic and disability conditions in the baseline.

The availability of informal care is divided into two dummy variables to capture whether the care is provided by sons or daughters. To measure whether gender of caregiver has an effect on a female elderly, an interactive dummy is added to the original model described in equation 2. After several rounds of calibration, we included in the final estimations a sub-

\footnotetext{
${ }^{3}$ It is important to highlight that one may run a similar specification controlling for baseline medical expenditures instead of a baseline individual's health. Clearly, medical expenditures may have an important causal link to health; yet, medical expenditure is an endogenous variable so adding it to the model will complicate the estimation. Adding previous health status would capture this effect since both variables are correlated. We tried several specifications with medical expenditures in the baseline year; however, we used a larger sample and therefore obtain more stable results when we used baseline health.
} 
sample of the control variables reported in Table 5. Since each of our dependent variables are dummies, logistic regressions are run to fit the data. In each case, we first run a naïve model (i.e., a model that includes the availability of informal care, gender of the elderly, and baseline health condition); then, we run a full model that includes the vector of control variables.

To assess the relevance of informal care provided by daughters, using the naive model, we predict the impact of informal care under two different scenarios: scenario 1 refers to a case where everyone in the sample has a female caregiver; scenario 2 refers to a case where both variables-male caregiver and female caregiver-are set up equal to zero.

Three strategies were implemented to test the sensitivity of the estimates to selection bias due to mortality. First, we estimated equation (3) using only the sample of live individuals; second, we included individuals who died and estimated the equation of interest by adding an additional category to the individual's description of health (i.e., for SRHS the category would be dying $=0,1=$ very bad, $2=$ bad, $3=$ good, $4=$ very good, $5=$ excellent). Last, we estimated two-part models where we first estimated the probability of dying and then, conditional on surviving, we estimated the probability of having a decline in health. Below, we discuss the results when one uses the sample of live individuals. The results from the other two alternatives did not differ from the results presented in this section. ${ }^{4}$

\section{RESULTS}

In this section we report the results from running the model described in equation (3). We first present results on the probability of dying and then present results for changes in health during the observed period, using as indicators SRHS, ADL, and IADL. Next, we look at caregivers' effect on the health of an elderly with specific chronic or disability conditions.

\section{Probability of Dying (Table 4)}

Table 4 shows the results of having informal care available in 2001 on the probability of dying between 2001 and 2003. Model 1 estimates indicate that informal care provided by sons reduces the probability of dying, but the effect is not significant at $p<0.05$. On the other hand, informal care that daughters provide reduces the probability of dying after controlling for gender of the elderly and initial health condition (ADL index). The estimate suggests that a daughter's care has a greater protective effect on the probability of dying than a son's care. This effect is statistically significant at $p<0.05$ and relevant in magnitude. These findings need to be interpreted with caution. In particular, these gender differences in the impact of informal care on an elderly person's health may be due to the fact that daughters provide higher quantity and quality of care than sons.

Using our simulation strategy described above, this study finds that having a female caregiver reduces the probability of dying from $0.066(0.081)^{5}$ to $0.0209(0.032)$. When one adds additional control variables, informal care from a female caregiver is still statistically

\footnotetext{
${ }_{5}^{4}$ These results are available from the authors upon request.

${ }^{5}$ The standard error for the sample prediction is in parenthesis.
} 
significant at $p<0.05$ (see Table 4). The magnitude of the effect of having a female caregiver is still relevant: approximately $3 \%$ points when one compares the two previous scenarios. In both models, the effect of having a female caregiver is greater than having a male caregiver.

According to the full model, an elderly female has a lower probability of dying than an elderly male; the probability of dying increases with age and decreases for married couples; however, the coefficient for married is not significant at $p<0.05$. Holding constant other variables, an increase in total net wealth reduces the probability of dying; this result is statistically significant only at $p<0.10$. Last, seniors with higher functional health (i.e., higher ADL) in 2001 were less likely to die during the study period.

\section{Self-Reported Health, ADL, and IADL (Table 5)}

Table 5 shows the logistic regression for the naïve model and the full model for SRHS, ADL, and IADL. According to Model 1, both daughters' and sons' informal care reduces the probability of a decline in SRHS; yet, the informal care that daughters provide has a greater impact than the care sons provide (see Table 5 ). In both cases, the availability of a caregiver is not statistically significant at $p<0.05$. When one predicts the impact of having a daughter as a caregiver, using Model 1 under scenario 1, the probability of having a decline in SRHS is $0.246(0.189)$; this probability equals $0.258(0.193)$ under scenario 2 .

According to the full model, having a daughter caregiver still has a protective effect on SRHS (i.e., negative coefficient). Nevertheless, the net effect is smaller than the one observed in the naïve model and is not significant at $p<0.05$.

In the case of ADL, having a daughter as caregiver in the baseline year did have a protective effect on changes in ADL during the following two years. In fact, an elderly person receiving informal care from daughters was less likely to report a decline in ADL during the study period. This result is significant in both models (see Table 5). The magnitude of the effect is moderate in both cases. The effect of sons as caregivers is not statistically significant and reverse in sign.

Table 5 also presents the results in the case of IADL. For the naïve model, having a daughter as a caregiver reduces the probability of having a decline in IADL during the next two years. The daughter coefficient $(0.497)$ is significant at $p<0.05$. The son variable has an opposite and smaller effect, yet it is not statistically significant at $p<0.05$. The result for the full model is similar in direction and size of the effect. As in the previous case, the effect of sons as caregivers is not statistically significant and is in an opposite direction.

Regarding the other variables, for all models, female elderly are more likely to report a decline in SRHS, ADL, and IADL. Yet the result is significant at $p<0.05$ only in the case of IADL (see Table 5). Having better baseline health increases the probability of having a reduction in health during the study period. This may be due to the fact that during elderly years, it is most likely that SRHS and functional indicators decline over a short period of time while seniors who report low health may be likely to adapt to the low health condition. Last, in all cases, higher total net wealth reduces the probability of having a decline in 
health. However, the coefficient is statistically significant at $p<0.05$ only in the case of the SRHS.

\section{Effect of Informal Care on an Elderly Person With a Specific Chronic or Disability Condition (Table 6)}

Early in this article, we argued that the impact of informal care on a senior's health happens throughout different channels. In particular, informal care helps the elderly manage chronic or disability conditions. Informal care helps the elderly adapt to the environment and reduces future accidents, or informal care provides support to the elderly in their daily lives, which in time improves their functional health. Therefore, individuals who report a chronic or a disability condition may benefit differently from informal care.

In this section, we explore whether informal care influences the health indicators of individuals who report one of the following conditions: hypertension, diabetes, arthritis, or a fall in the last 12 months. Table 6 summarizes these results on the probability of dying.

Among seniors who reported hypertension in 2001, those who had a female caregiver were less likely to die, even after controlling for demographic, socioeconomic, and baseline health conditions (see Table 6). This effect is significant at $p<0.05$. In addition, among seniors with hypertension, the availability of a son as caregiver does not influence the change in seniors' health from 2001 to 2003 or the probability of surviving.

When one looks at seniors with diabetes, a similar effect is found. The availability of a female caregiver reduces the probability of dying during the study period $(p<0.05)$. As before, the availability of a son as caregiver does not influence changes in health among seniors with diabetes.

In the case of disability conditions, it is found that informal care from a daughter or a son does not influence changes in health from 2001 and 2003. In fact, as shown in Table 6, among seniors with arthritis or among those who had fallen during the past 12 months, having informal care does not influence their survival rate or the decline in health between both years.

In sum, informal care provided by daughters influences the probability of dying among seniors who report hypertension and diabetes. The results are less robust when one looks at the changes in functional health among these elderly. According to this study, care that sons provide seems not to be effective among seniors. This finding should be interpreted with caution since the lack of significance may be due to sample characteristics rather than a true causal effect. Nevertheless, this result may be consistent with the gender of parent hypothesis since more women than men had these conditions in the baseline year. Finally, the results indicate that among those with disability conditions (i.e., arthritis and fall), informal care is less effective in reducing seniors' probability of dying or in preventing the decline in their functional health from 2001 to 2003. 


\section{DISCUSSION}

This article provides new evidence on the effect of informal care on elderly health. While empirical findings suggest a trade-off between informal care and medical expenditures, the consequences of informal care on elderly health have been less documented. The aim of this paper has been not to identify the causal pathways between informal care and elderly health. Instead, the goal has been to explore whether there is a link between informal care and changes over time in health outcomes. The MHAS database provides a unique opportunity to answer this question.

The results indicate sizable gains of informal care in terms of reducing the probability of dying - even after controlling for living conditions, marital status, and baseline health. Informal care helps elderly parents at the margin to prolong their lives. The results are particularly important when one looks at patients with specific chronic conditions such as hypertension and diabetes. Caregivers can help elderly people manage their chronic conditions better and improve the environment where they live, which in time increases their survival. Alternatively, caregivers may help disabled elderly parents conduct their daily activities and prolong their lives. Clearly, the monetary benefits of reducing the probability of dying need to be taken into consideration when policymakers conduct cost/benefit analyses of informal care.

The findings suggest that after controlling for baseline health, the protective effect of informal care in terms of self-reported health is considerably low-regardless of the caregiver's gender. As expected, controlling for baseline health conditions, care provided by daughters reduced the probability of having a decline in functional activities of the elderly (ADL and IADL). These gender differences in the impact of informal care on elderly health may be due to the fact that daughters provided higher quantity and quality of care than sons.

Social policy reforms must be implemented soon to deal with Mexico's rapidly aging population. If necessary changes are made now, daunting and costly tasks in the future may not be necessary. This study enriches our understanding of family caregivers for the elderly and suggests an additional benefit in terms of health. This benefit needs to be weighed to evaluate policy alternatives regarding informal care to complement the expansion of both direct medical care and pension benefits to support and protect the elderly population. These benefits can be of particular relevance to reduce the social costs associated with treatment of costly chronic conditions such as diabetes and hypertension among the elderly population.

Finally, these findings should be taken with caution. Since the MHAS includes only two waves of data, the authors are not able to identify how changes in caregiver availability influence changes in health over time and are not able to identify the potential dynamic feedback between elderly health and individuals' decision to provide informal care. Moreover, unfortunately, we cannot disentangle the channels through which informal care affects elderly health. Further research in this direction needs to be done. Last, this research did not compare the relative health benefits of informal and formal care, nor did it test the cost-effectiveness of either. We believe, however, that this research provides further evidence to conduct a more complete analysis. 


\section{Acknowledgments}

We thank Brian Brennan for excellent research assistance during the development of this project. We also express our gratitude to two anonymous reviewers and the editor of the journal for valuable comments and suggestions. The authors take sole responsibility for any remaining errors. We also thank the Inter-American Development Bank, Gender and Diversity Unit, for the financial support provided for this investigation. Last, we acknowledge the principal investigators who participated in the National Mexican Health and Aging Study.

\section{References}

1. Wong R, Diaz JJ, Higgins M. Health care use among elderly Mexicans in the United States and Mexico: The role of health insurance. Res Aging. 2006; 28:393.

2. Van den Berg, B.; Ferrer-i-Carbonell, A. Working Paper. Amsterdam Institute for Advanced Labor Studies; The Netherlands: 2004. The well-being of informal caregivers: A monetary valuation of informal care.

3. Pinquart M, Sorensen S. Gender differences in caregiver stressors, social resources, and health: An updated meta-analysis. J Gerontol B Psychol Sci Soc Sci. 2006; 61(1):33-45.

4. Donelan K, Hill CA, Hoffman C. Challenged to care: Informal caregivers in a changing health system. Health Aff (Millwood). 2002; 21(4):222-231. [PubMed: 12117133]

5 . Finley NJ. Theories of family labor as applied to gender differences in caregiving for elderly parents. J Marriage Fam. 1989; 51(1):79-86.

6. Lee GR, Dwyer JW, Cowart RT. Gender differences in parent care: Demographic factors and samegender preferences. J Gerontol. 1993; 48(1):9-16.

7. Van Houtven C, Norton E. Informal care and Medicare expenditures: Testing for heterogeneous treatment effects. J Health Econ. 2008; 27:134-156. [PubMed: 17462764]

8. Clark NN. Management of chronic disease by patients. Ann Rev Pub Health. 2003; 24:289-313. [PubMed: 12415147]

9. Gallant M, Spitze G, Prohaska T. Help or hindrance? How family and friends influence chronic illness self-management among older adults. Res Aging. 2007; 29:375.

10. Antonucci, T.; Jackson, J. Social support, interpersonal efficacy, and health: A life course perspective. In: Cartensen, LL.; Edelstein, BA., editors. Handbook of Clinical Gerontology. Pergamon Press; Elmsford, New York: 1987.

11. Gallant MP. The influence of social support on chronic illness self-management: A review and directions for research. Health Educ Behav. 2003; 30:170-195. [PubMed: 12693522]

12. Neal M, Ingersoll-Dayton B, Starrels M. Gender and relationship differences in caregiving patterns and consequences among employed caregivers. Gerontologist. 1997; 37(6):804-816. [PubMed: 9432997]

13. Iacovou, M. The living arrangements of elderly Europeans. Institute for Social and Economic Research; Feb. 2000 p. 1-35.

14. Sarkisian N, Gerstel N. Explaining the gender gap in help to parents: The importance of employment. J Marriage Fam. 2004; 66(2):431-451.

15. Heidemann B, Stern S. Strategic play among family members when making long-term care decisions. J Econ Behav Organ. 1999; 40(1):49-57.

16. Enger M, Stern S. Long-term care and family bargaining. Int Econ Rev. 2002; 43(1):73-114.

17. Stern S. Estimating family long-term care decision in the presence of endogenous child characteristics. J Hum Resour. 1995; 30(3):551-580.

18. Pagan J, Puig A, Soldo B. Health insurance coverage and the use of preventative services by Mexican adults. Health Econ. 2007; 10:1002.

19. Deeg D, Kriegsman D. Concepts of self-related health: Specifying the gender difference in mortality risk. Gerontologist. 2003; 43(3):376-386. [PubMed: 12810902]

20. Strauss J, Getler P, Rahman O, et al. Gender and the life-cycle differentials in the patterns and determinants of adult health. J Hum Resour. 1993; 28(4):791-837. 
21. Saab, P. Transferencias informales de apoyo de los adultos mayores en America Latina y el Caribe: Estudio comparativo de encuestas. Society for the Advancement of Behavioral Economics; 2002. p. $175-218$.

22. Hedeker, D.; Gibbons, RD. Longitudinal Data Analysis. John Wiley \& Sons, Inc; Hoboken, New Jersey: 2007. 


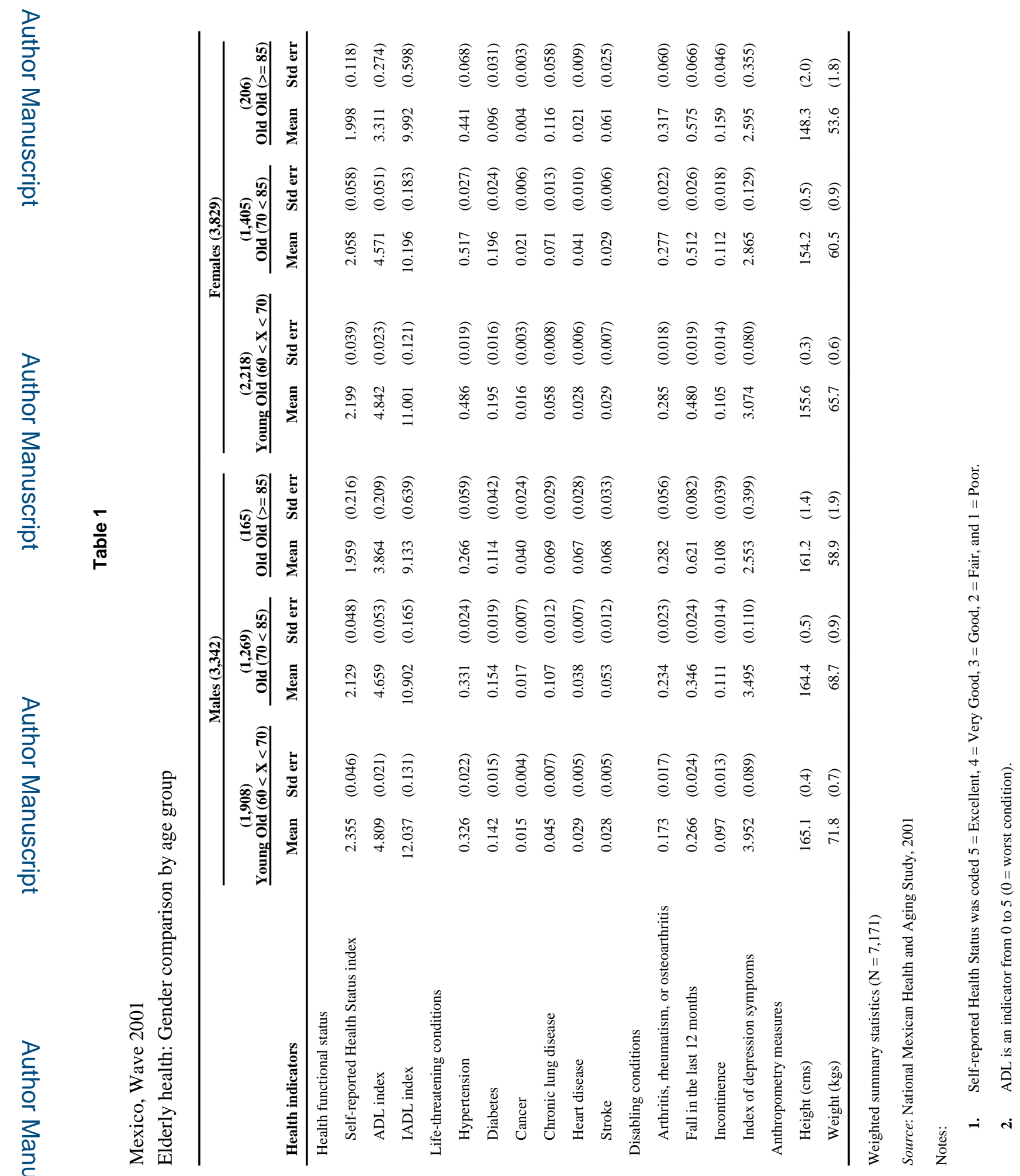




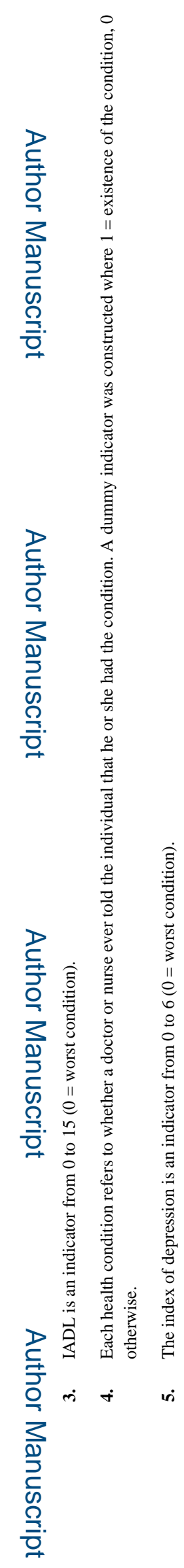

Int J Health Serv. Author manuscript; available in PMC 2015 March 25. 


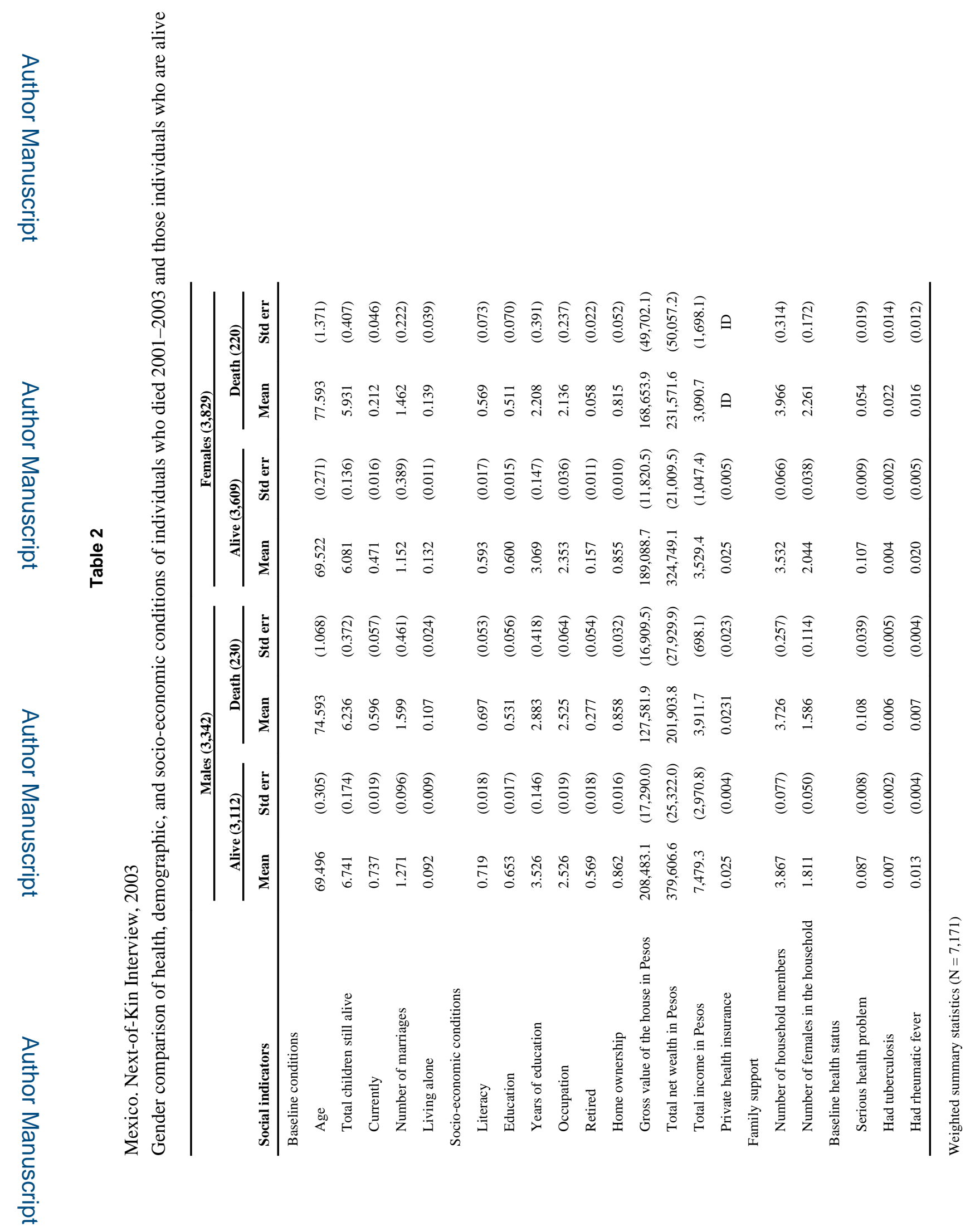

Int J Health Serv. Author manuscript; available in PMC 2015 March 25. 

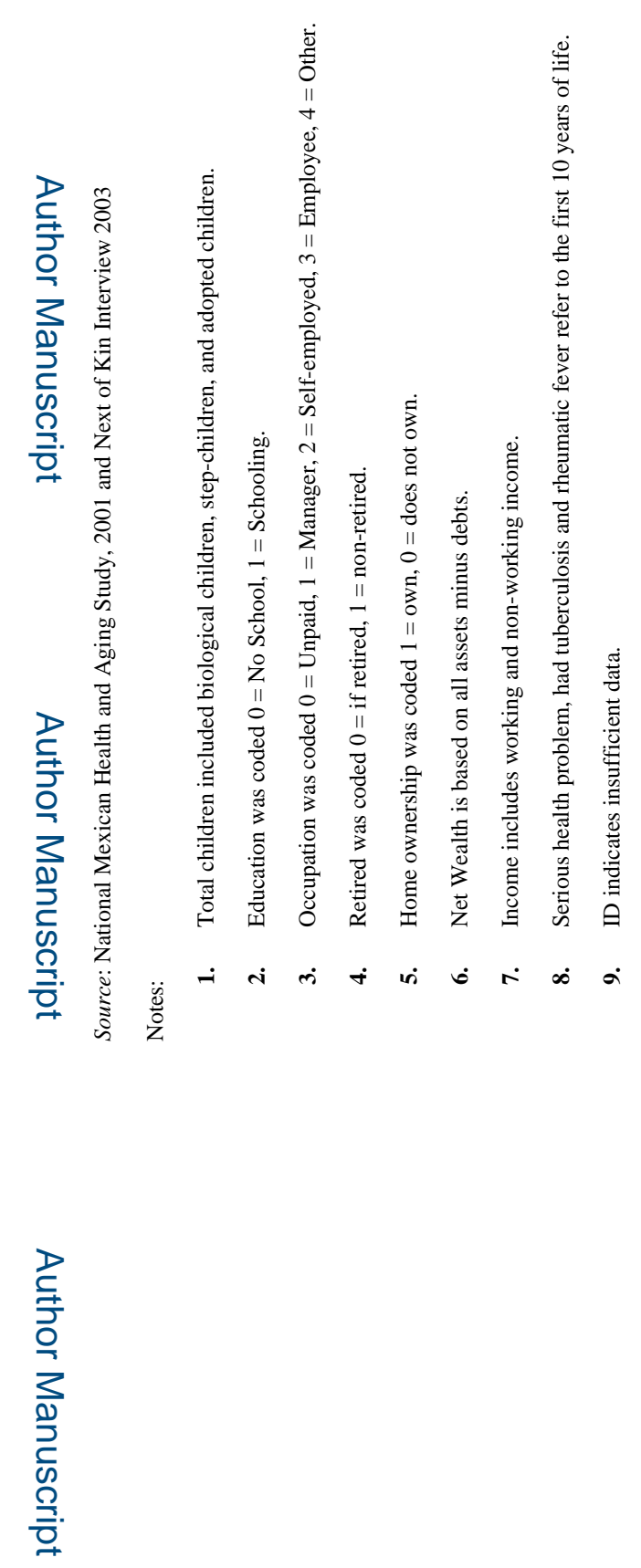

로을

Int J Health Serv. Author manuscript; available in PMC 2015 March 25. 


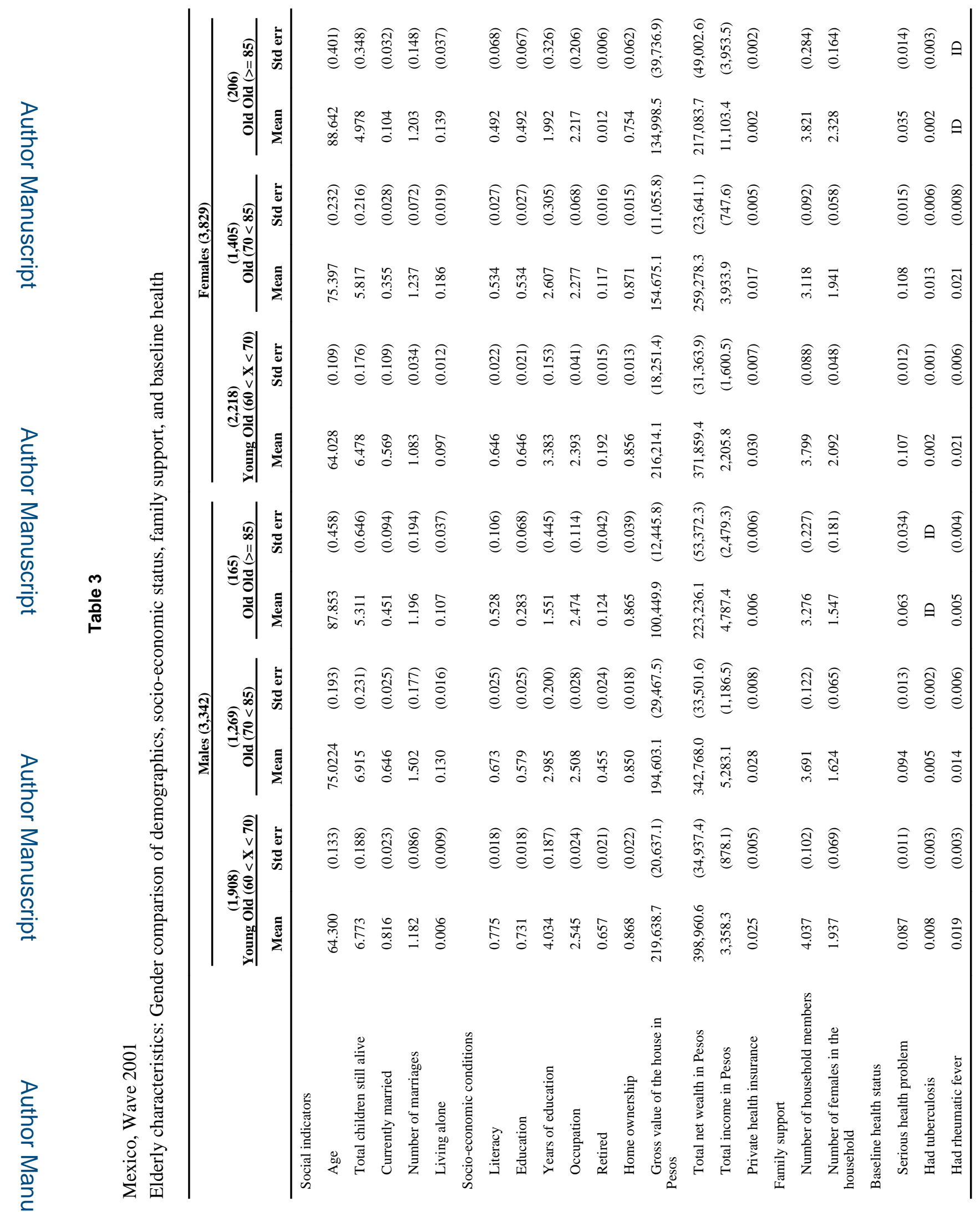

Int J Health Serv. Author manuscript; available in PMC 2015 March 25. 

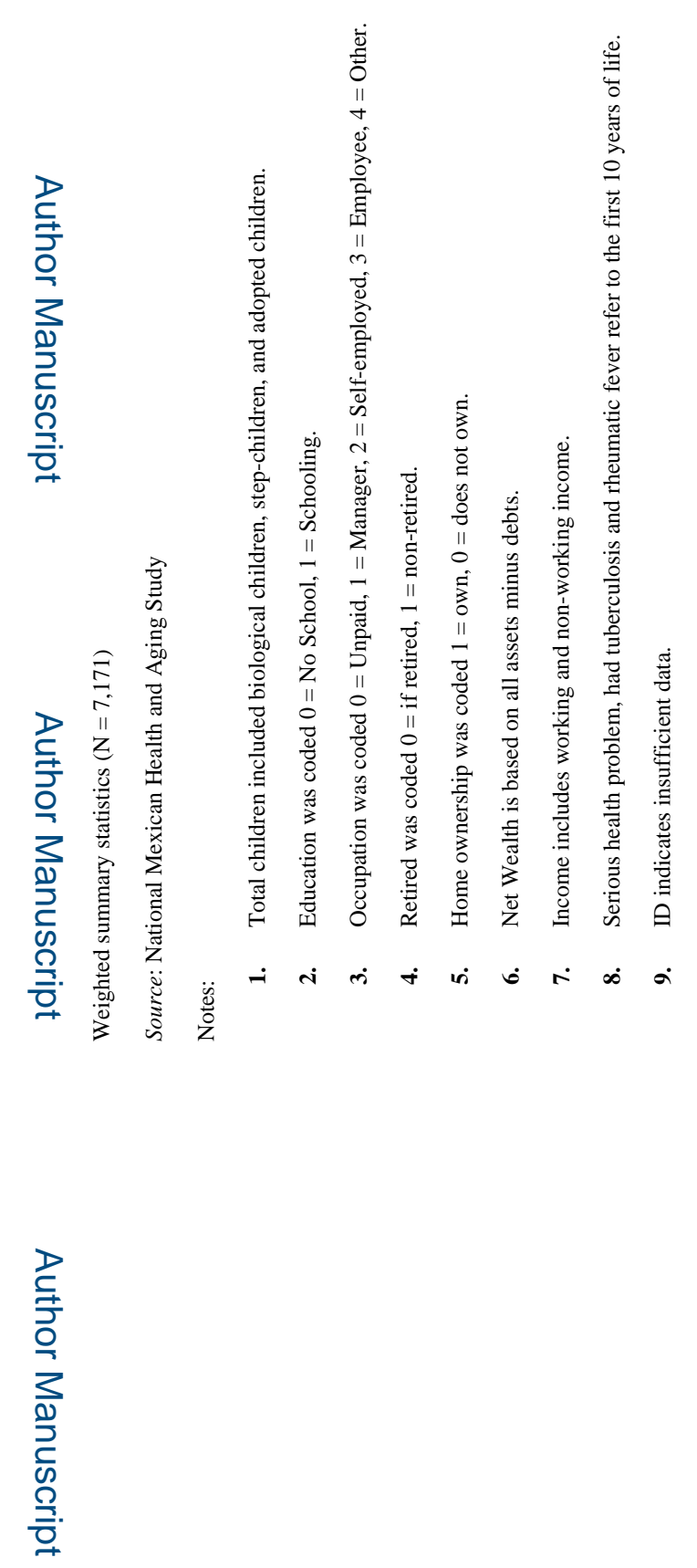

로을

Int J Health Serv. Author manuscript; available in PMC 2015 March 25. 


\section{Table 4}

\section{Mexico}

Logistic estimates of the effect of informal care on the elderly's death Dependent variable: Dying between 2001 and 2003 Weighted statistics

\begin{tabular}{|c|c|c|c|c|}
\hline \multirow{2}{*}{$\begin{array}{l}\text { Variables } \\
\text { Independent variables (2001) }\end{array}$} & \multicolumn{4}{|c|}{ Probability of dying between 2001 and 2003} \\
\hline & \multicolumn{2}{|c|}{ Model 1} & \multicolumn{2}{|c|}{ Full model } \\
\hline Son caregiver $(1=$ if available $)$ & -0.385 & $(0.623)$ & 0.341 & $(0.586)$ \\
\hline Daughter caregiver ( 1 = if available) & -1.357 & $(0.513)$ & -0.912 & (0.049) \\
\hline \multicolumn{5}{|l|}{ Control variables (2001) } \\
\hline Female & -0.546 & $(0.192)$ & -0.527 & $(0.227)$ \\
\hline Age & & & 0.045 & (0.013) \\
\hline Currently married ( 1 = currently married $)$ & & & -0.267 & $(0.244)$ \\
\hline Living alone ( 1 = living alone $)$ & & & 0.161 & $(0.292)$ \\
\hline Literacy & & & 0.205 & $(0.233)$ \\
\hline Home ownership $(1=$ owner $)$ & & & 0.025 & $(0.301)$ \\
\hline Total net wealth in Pesos & & & -0.0001 & $(0.000)$ \\
\hline \multicolumn{5}{|l|}{ Health status (2001) } \\
\hline ADL index & -0.619 & $(0.058)$ & -0.515 & (0.064) \\
\hline Intercept & 0.374 & $(0.283)$ & -3.219 & (1.085) \\
\hline $\mathrm{N}$ & 6,811 & & 6,811 & \\
\hline
\end{tabular}

Source: National Museum Health and Aging Study, 2001 and Next-of-Kin Interviews.
a. Estimates in bold are significant at the 5\% level.
b. Standard errors in parenthesis.
c. Net wealth is based on all assets minus debts. 

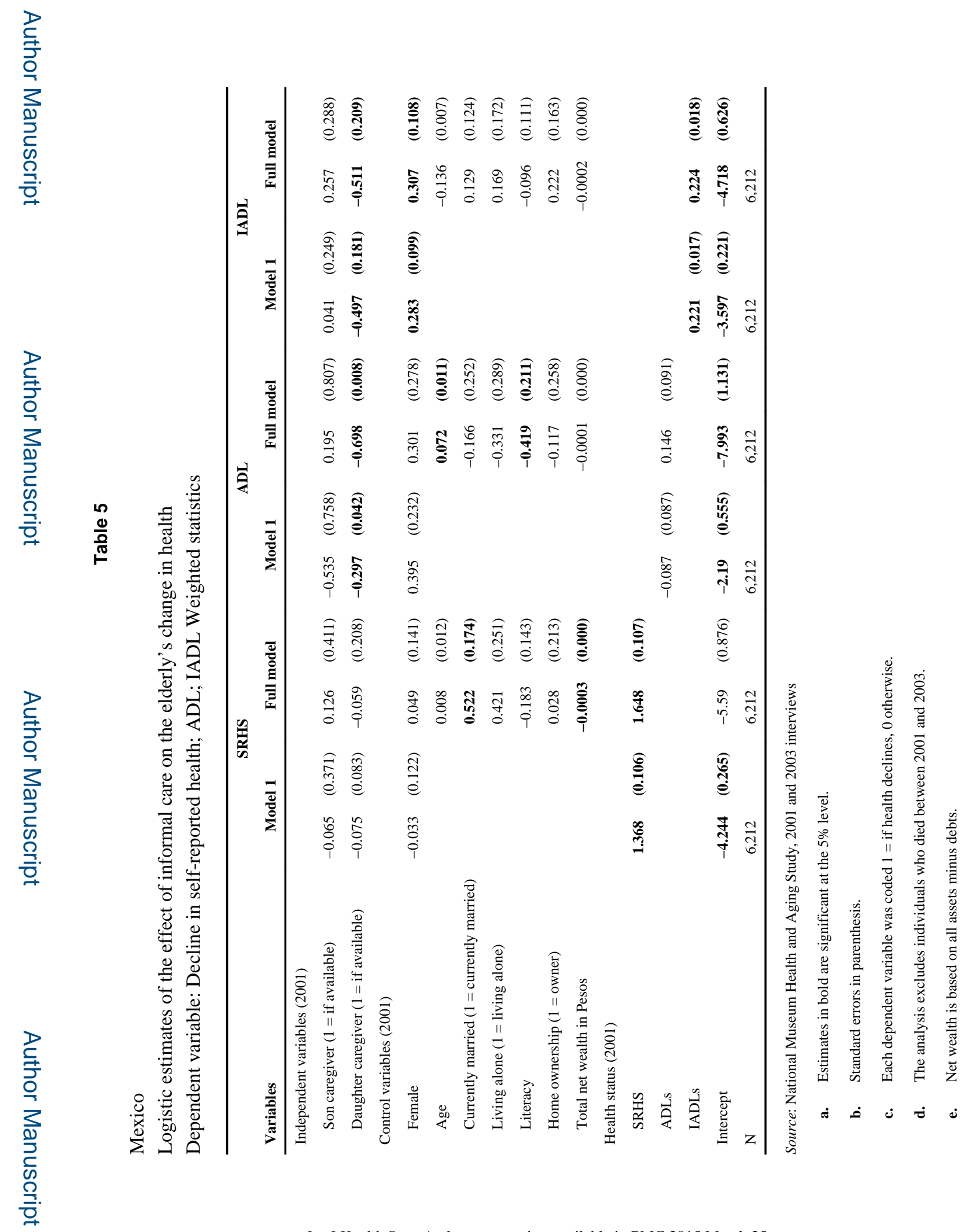

Int J Health Serv. Author manuscript; available in PMC 2015 March 25. 


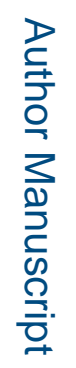

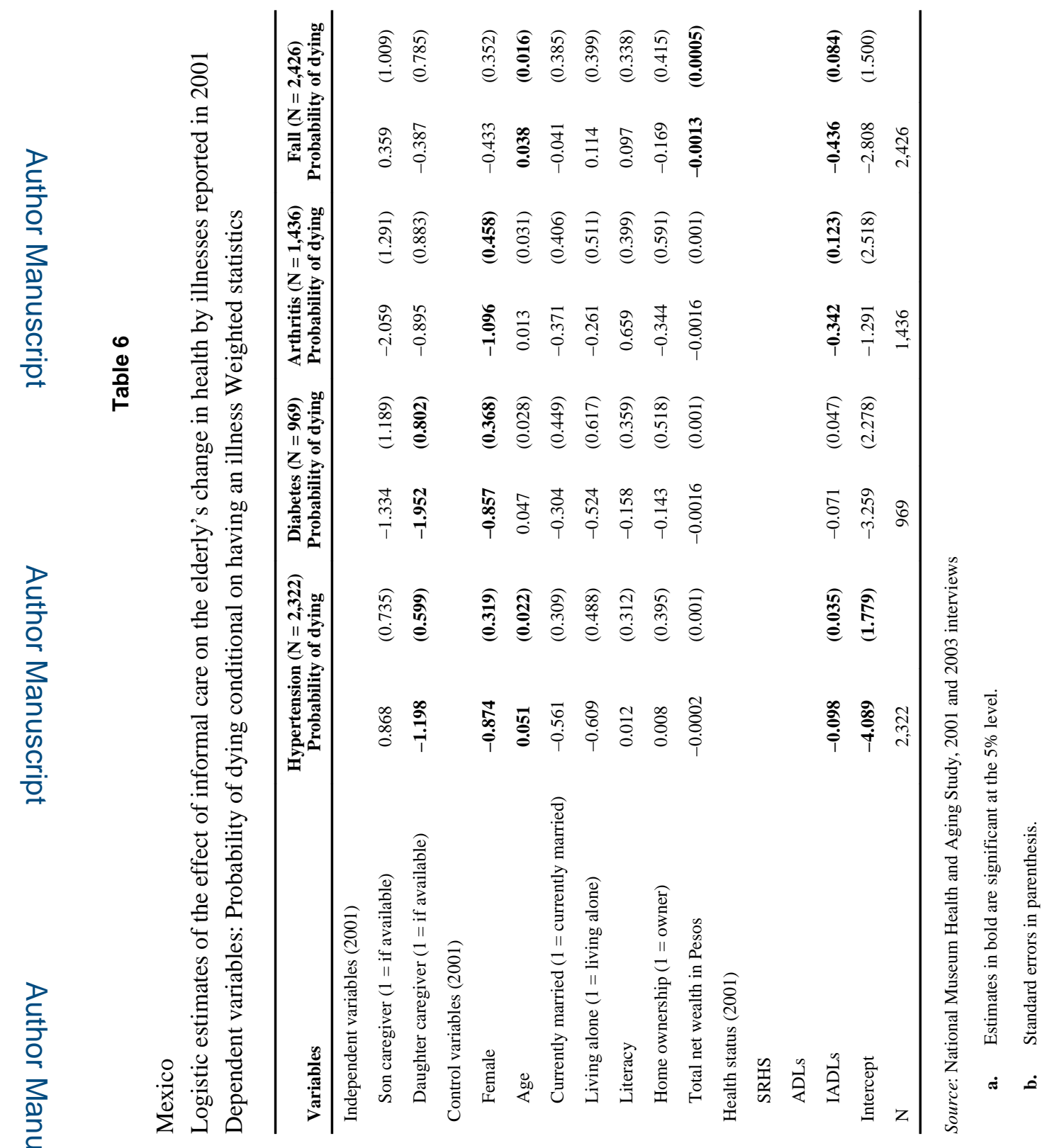

Int J Health Serv. Author manuscript; available in PMC 2015 March 25. 